\title{
A Design for a Manufacturing-Constrained Off-Axis Four-Mirror Reflective System
}

\author{
Ruoxin Liu, Zexiao Li, Yiting Duan and Fengzhou Fang *(1) \\ State Key Laboratory of Precision Measuring Technology \& Instruments, Centre of MicroNano Manufacturing \\ Technology-MNMT, Tianjin University, Tianjin 300072, China; ruoxin_liu@tju.edu.cn (R.L.); \\ zexiaoli@tju.edu.cn (Z.L.); ytduan@tju.edu.cn (Y.D.) \\ * Correspondence: fzfang@tju.edu.cn
}

Received: 28 June 2020; Accepted: 30 July 2020; Published: 4 August 2020

\begin{abstract}
Off-axis reflective optical systems find wide applications in various industries, but the related manufacturing issues have not been well considered in the design process. This paper proposed a design method for cylindrical reflective systems considering manufacturing constraints to facilitate ultra-precision raster milling. An appropriate index to evaluate manufacturing constraints is established. The optimization solution is implemented for the objective function composed of primary aberration coefficients with weights and constraint conditions of the structural configuration by introducing the genetic algorithm. The four-mirror initial structure with a good imaging quality and a special structural configuration is then obtained. The method's feasibility is validated by designing an off-axis four-mirror afocal system with an entrance pupil diameter of $170 \mathrm{~mm}$, a field of view of $3^{\circ} \times 3^{\circ}$ and a compression ratio of five times. All mirrors in the system are designed to be distributed along a cylinder.
\end{abstract}

Keywords: mirror system design; optical fabrication; constrained optimization

\section{Introduction}

Reflective optical systems have attracted much interest because of their extensive use in many fields such as space optical systems [1] and infrared scanning systems [2,3]. Due to the advantages of the reflective optical system such as being free of chromatic aberrations, a foldable optical path, a large aperture, insensitive to temperature and pressure changes, etc., more attention has been paid to the design of the reflective systems than to the refractive systems. In addition, making the field of view (FOV) off-axis can also solve the obstruction problem in reflective systems [4]. Among them, the four-mirror system can obtain a good imaging quality, a compact structure, a large FOV, and a large aperture because of its higher design degrees of freedom.

With the development of the ultra-precision machining system, it is possible to manufacture reflective optical systems with complex surfaces such as aspheric surfaces and freeform surfaces [5-7]. The reflective optical systems usually go through a series of processes (e.g., design, machining, assembling, etc.) before being put into use. However, most of the current design methods focus only on how to improve the optical performance of these systems, while the manufacturing issues are not well considered in the design process. The consequence is that the optical system with complex surfaces is usually difficulty to achieve in machining and assembling, even if it may satisfy the optical requirements in theory. This reduces the practicability of the designed optical system. Introducing manufacturing constraints into the design process helps to simplify the machining and assembling process. Meng et al. developed an off-axis three-mirror system with the primary mirror and tertiary mirror integrated on a single substrate, in which the number of mirrors that need optical alignment is reduced from three to two [8]. Li et al. proposed an alignment-free manufacturing approach to 
machine the off-axis multi-reflective system, in which the measurement methods were also established to evaluate optical performance of the integrated system [9]. Zheng et al. proposed the concept of the reference surface to limit the position of each mirror in an off-axis three-mirror anastigmat (TMA) optical system [10]. The reference surface theory requires that the mirror's surface must be close enough to the reference surface to make the machining process more accessible. The shape of the reference surface depends on the machining method: for the turning and milling process, the reference surface are the symmetric rotational surface and the extended surface, respectively [11-13]. A cylindrical reference surface also helps to make the machining process more effective. When all mirror surfaces in a reflective optical system are close to the same cylindrical surface, the milling process will be greatly simplified, and the optical alignment caused by the asymmetry of the freeform surface can also be avoided. Therefore, the similarity between the mirror's surfaces and the reference surface should be considered at the level of design.

At present, the mainstream optical design software adopts the damped least square (DLS) method as the optimization algorithm $[14,15]$. This algorithm is a local optimization algorithm, which is easy to fall into the local minimum value in the optimization process, so the final design result is often the local optimal solution close to the initial point rather than the global optimal solution. Therefore, the existing optical design software depends heavily on the selection of the initial structure. In other words, the selection of a reasonable initial structure is a critical step in the optical system design. Generally, in order to design an optical system that meets the requirements, it is necessary to find the initial structure parameters from the existing references and then optimize the initial structure. However, for some optical systems with special structural configurations, this method may not be applicable. To meet different design requirements, the structure of the optical system cannot rely on the existing initial structure excessively. Based on the genetic algorithm (GA), this paper presents a method to automatically calculate the initial structural parameters that satisfy the manufacturing constraints.

GA is a highly parallel, random and adaptive global optimization algorithm proposed by Holland in 1975, which is based on Darwin's theory of "survival of the fittest" [16,17]. The algorithm iteratively optimizes the objective function by relying on biologically inspired operators such as mutation, crossover and selection. GA has been widely used in the optimization of optical systems [18-21], such as diffractive optical elements [22], the deformable mirror in adaptive optical systems, the wavefront coding system [23,24], and the design of the optical system with large FOV. In this paper, GA is used to find practicable initial structures of reflective optical systems with special requirements.

\section{Working Principle}

\subsection{Manufacturing Constraints Analysis for Four-Mirror System}

Manufacturing constraints in optical systems depend on practical manufacturing methods. Figure 1a demonstrates a computer integrated manufacturing system for an off-axis four-mirror reflective optical system based on raster milling.

In order to avoid the time-consuming machining process and the tedious assembly process before the system is put into use, all the mirror blanks can be assembled according to the design position, and then all the mirrors can be processed synchronously by raster milling.

In this method of processing, all mirrors are free of disassembly and assembly, in which the position accuracy of the mirrors depends only on the machine tool. Compared with the traditional method, there is no need to process the locating surface so that the alignment error can be avoided, and the system can be put into use after the processing is completed. An ultra-precision machine tool has a high motion accuracy, which ensures the excellent position accuracy between the mirrors. Therefore, the good optical performance of the whole system is ensured.

The curvature radii of the mirror surfaces in the system should be greater than the rotational radius of the tools, and all the mirror surfaces should be close to a cylindrical reference surface, as shown in 
Figure $1 \mathrm{~b}$. These manufacturing constraints should be reflected in the design process of the system to ensure a smooth machining process.

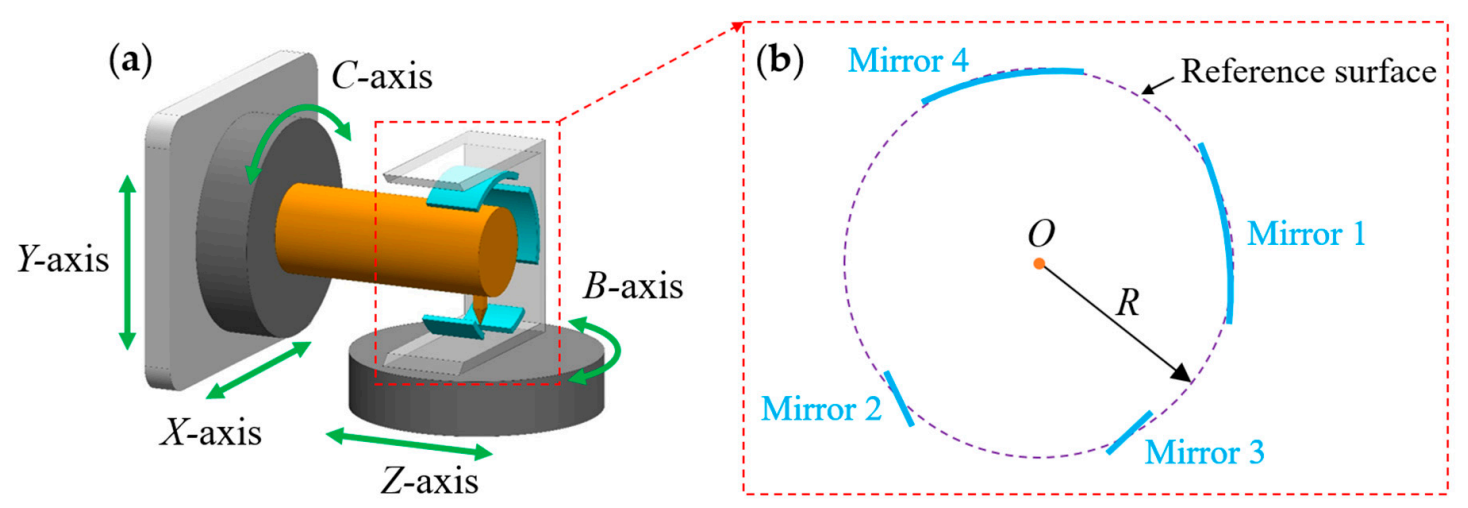

Figure 1. (a) Machining configuration for the four-mirror system. (b) The geometric relationship between the mirrors' surfaces and the reference surface. In the figure, $O$ is the center of the reference surface and $R$ is the radius of the reference surface.

In practice, there is always a deviation between the mirror and the reference surface. For one mirror in this system, the region between the mirror's surface and the reference surface is called the deviation region, as shown in Figure 2.

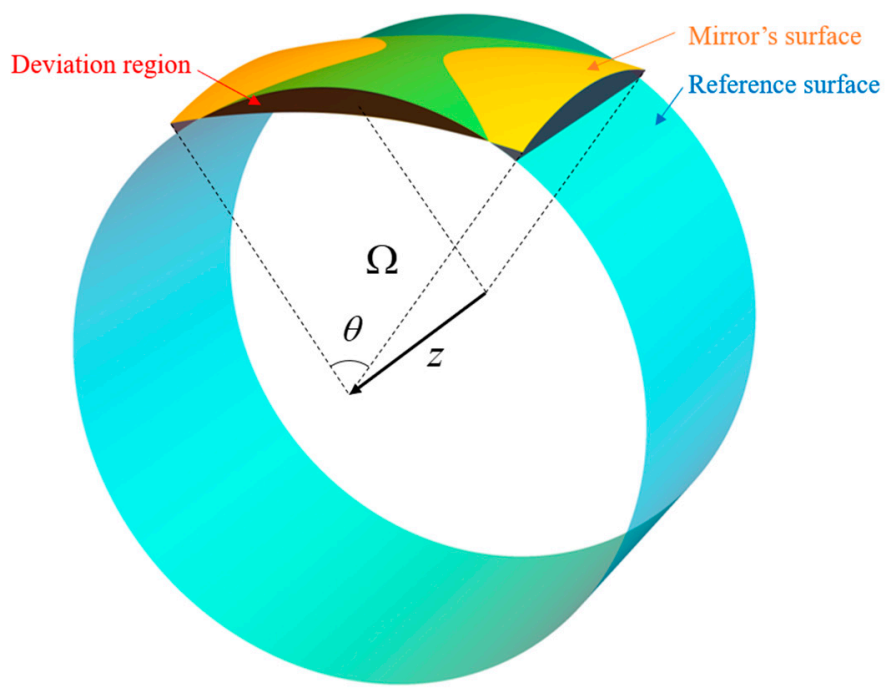

Figure 2. The deviation region between the mirror's surface and the reference surface. In the figure, $\Omega$ is the integral region and $\theta$ is the angle formed by the mirror's edge and the Z-axis of the cylindrical coordinate system.

To quantify this deviation, the index called the average deviation from the reference surface (ADRS), $\sigma_{\mathrm{ADRS}}$, is defined to characterize the average deviation between the mirrors' surface and the reference surface in the design,

$$
\sigma_{\mathrm{ADRS}}=\frac{1}{\Omega} \int_{\Omega}|S-R| d \Omega,
$$


where $S$ is the equation of the mirror's surface in the cylindrical coordinate system and $R$ is the radius of the reference surface. $\Omega$ is the integral region, which has the following differential form:

$$
d \Omega=d \theta d z
$$

where $\theta$ is the angle formed by the mirror's edge and the Z-axis of the cylindrical coordinate system.

In the raster milling process, the deviation region of a cross-section perpendicular to the tool's rotation direction should also be considered, as shown in Figure 3a.

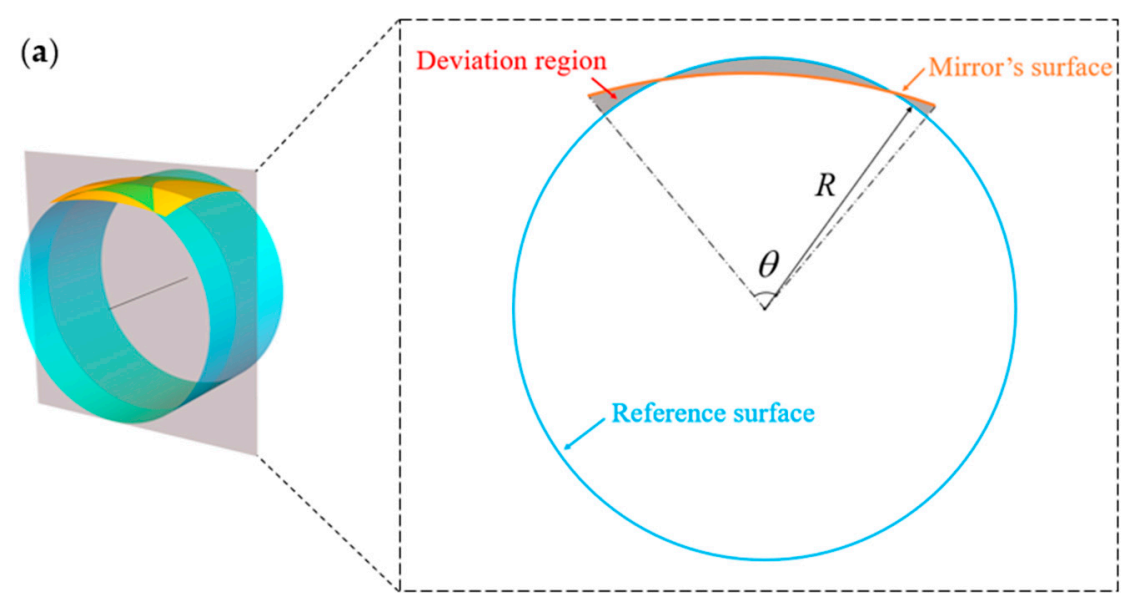

(b)
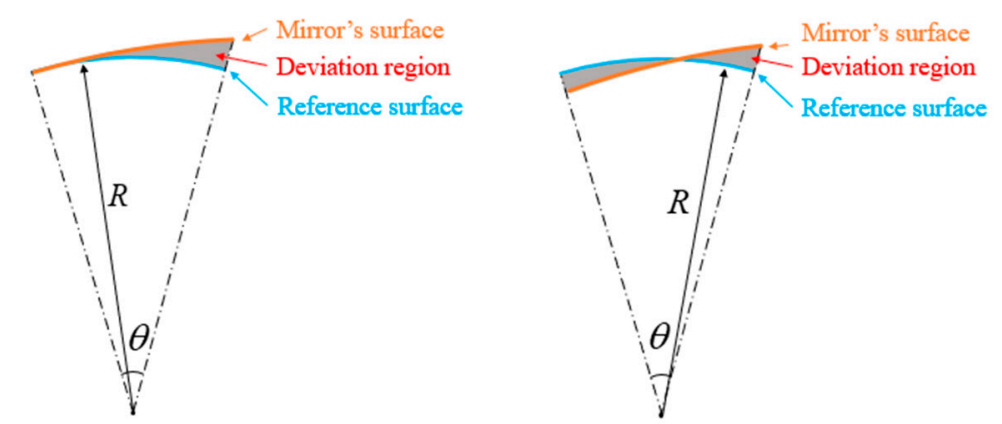

Figure 3. (a) The deviation region between the mirror's surface and the reference surface in a cross-section. (b) Other possible positional relationships of the mirror and the reference surface.

In a cross-section of this structure, Equation (1) can be rewritten as

$$
\sigma_{\mathrm{ADRS}}=\frac{1}{\theta} \int|\rho-R| d \theta,
$$

where $\theta$ is the central angle of the mirror, $\rho$ is the equation of the mirror's surface in the polar coordinate system and $R$ is the radius of the reference surface. Equation (3) also applies to other possible positional relationships of the mirror and the reference surface, as shown in Figure $3 b$. This index can effectively reflect the deviation degree regardless of the different positions and sizes of mirrors. Meanwhile, the maximum deviation from the reference surface of each mirror also needs to be considered.

In order to make this machining method easier to achieve, it is necessary to plan the tool's radius of gyration and tool spindle path in the design stage. For a cross-section perpendicular to the tool's rotation direction in the structure, draw the equidistance lines of the mirror's surface $L_{1}\left(R_{t}\right), L_{2}\left(R_{t}\right)$, $L_{3}\left(R_{t}\right)$, and $L_{4}\left(R_{t}\right)$ toward the center of the reference surface. The distance between the ends of the equidistant lines of adjacent mirrors is denoted by $d_{i}\left(R_{t}\right)(i=1,2,3,4)$. The equidistant line parameter $d$ is equal to the tool's radius of gyration $R_{t}$, as shown in Figure 4 . 


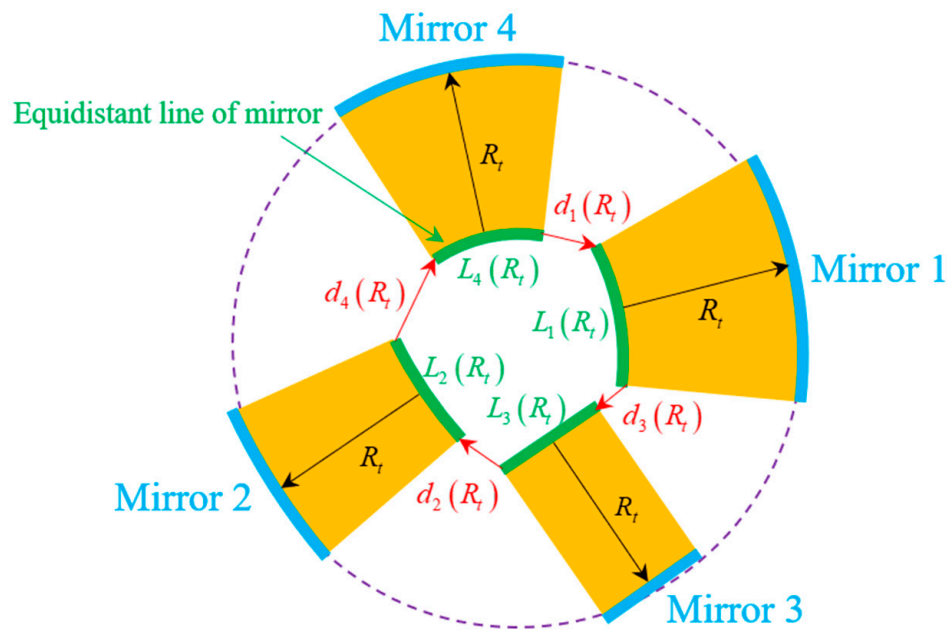

Figure 4. The tool turning radius and tool path in the structure. In the figure, $L_{i}\left(R_{t}\right)$ is the equidistance lines of the mirror's surface, $d_{i}\left(R_{t}\right)$ is the distance between the ends of the equidistant lines of adjacent mirrors and $R_{t}$ is the tool's radius of gyration.

The moving range of the tool spindle in the X-Y plane decreases as the tool's radius of gyration $R_{t}$ increases. When $\sum_{i=1}^{4} d_{i}\left(R_{t}\right)$ reaches the minimum value, the tool's radius of gyration $R_{t}$ reaches its maximum $R_{t \max }$ as shown in the following equation:

$$
\exists R_{t}=R_{t \max }, R_{t \max }=\underset{R_{t}}{\operatorname{argmin}} \sum_{i=1}^{4} d_{i}\left(R_{t}\right) .
$$

When the maximum radius of gyration $R_{t \max }$ is obtained, the tool spindle path $C$ is the closed path surrounded by $L_{i}\left(R_{t \max }\right)$ and $d_{i}\left(R_{t \max }\right)$, which is calculated by the following formula:

$$
C=\sum_{i=1}^{4}\left(L_{i}\left(R_{t \max }\right)+d_{i}\left(R_{t \max }\right)\right) .
$$

\subsection{Aberration Analysis for Four-Mirror System}

According to the particular constraints proposed by the previous section, we chose a four-mirror structure that contains a plane mirror. The plane mirror can fold the beam path to meet the special manufacturing method mentioned above and also provides the degree of freedom for the final aberration correction. Figure 5 shows the layout of the four-mirror reflective system.

This system is composed of three curved mirrors and one plane mirror: a primary mirror $\left(M_{1}\right)$, a secondary mirror $\left(M_{2}\right)$, a tertiary mirror $\left(M_{3}\right)$ and a quaternary mirror $\left(M_{4}\right)$, in which $M_{3}$ is the plane mirror tilted at an angle of $\theta_{3}$. The conic coefficients of $M_{1}, M_{2}$, and $M_{4}$ in this structure are $k_{1}$, $k_{2}$, and $k_{4}$. The incident ray heights of $M_{1}, M_{2}$, and $M_{4}$ are $h_{1}, h_{2}$ and $h_{4} \cdot l_{i}(i=2,4)$ and $l_{i}^{\prime}(i=2,4)$ are the object distances and the image distances of $M_{2}$ and $M_{4}$.

The obscure ratios of $M_{2}$ to $M_{1}$ and $M_{4}$ to $M_{2}$ are $\alpha_{1}$ and $\alpha_{2}$, respectively, and the magnification ratios of $M_{2}$ and $M_{4}$ are $\beta_{1}$ and $\beta_{2}$, respectively. They are defined as follows:

$$
\left\{\begin{array}{l}
\alpha_{1}=\frac{l_{2}}{f^{\prime}{ }_{1}} \approx \frac{h_{2}}{h_{1}}, \beta_{1}=\frac{l_{2}^{\prime}}{l_{2}}=\frac{u_{2}}{u_{2}} \\
\alpha_{2}=\frac{l_{4}}{l^{\prime}{ }_{2}} \approx \frac{h_{4}}{h_{2}}, \beta_{2}=\frac{l_{4}^{\prime}}{l_{4}}=\frac{u_{4}}{u_{4}^{\prime}}
\end{array} .\right.
$$


According to the paraxial optical theory [25], the obscure ratios and magnification ratios defined by the Equation (6), the curvature radius of the mirror and the distance between the mirror $i$ and the mirror $i+1$ can be deduced as follows:

$$
\begin{gathered}
\left\{\begin{array}{l}
R_{1}=\frac{2 f^{\prime}}{\beta_{1} \beta_{2}} \\
R_{2}=\frac{2 \alpha_{1} f^{\prime}}{\beta_{2}\left(1+\beta_{1}\right)} \\
R_{4}=\frac{2 \alpha_{1} \alpha_{2} f^{\prime}}{1+\beta_{2}}
\end{array}\right. \\
\left\{\begin{array}{l}
d_{1}=\frac{\left(1-\alpha_{1}\right) f^{\prime}}{\beta_{1} \beta_{2}} \\
d_{2}+d_{3}=\frac{\alpha_{1}\left(1-\alpha_{2}\right) f^{\prime}}{\beta_{2}} \\
d_{4}=\alpha_{1} \alpha_{2} f^{\prime}
\end{array}\right.
\end{gathered}
$$

where $f^{\prime}$ is the focal length of the four-mirror optical system.

Based on the primary aberration theory [26], we can obtain the third-order aberrations expressed in terms of the structure parameters $\alpha_{1}, \alpha_{2}, \beta_{1}, \beta_{2}, k_{1}, k_{2}$ and $k_{4}$ [27]. Due to space limitation, the implicit functions are as follows:

$$
\left\{\begin{array}{l}
S_{1}=S_{1}\left(\alpha_{i}, \beta_{i}, k_{i}\right) \\
S_{2}=S_{2}\left(\alpha_{i}, \beta_{i}, k_{i}\right) \\
S_{3}=S_{3}\left(\alpha_{i}, \beta_{i}, k_{i}\right) \\
S_{4}=S_{4}\left(\alpha_{i}, \beta_{i}, k_{i}\right) \\
S_{5}=S_{5}\left(\alpha_{i}, \beta_{i}, k_{i}\right)
\end{array}\right.
$$

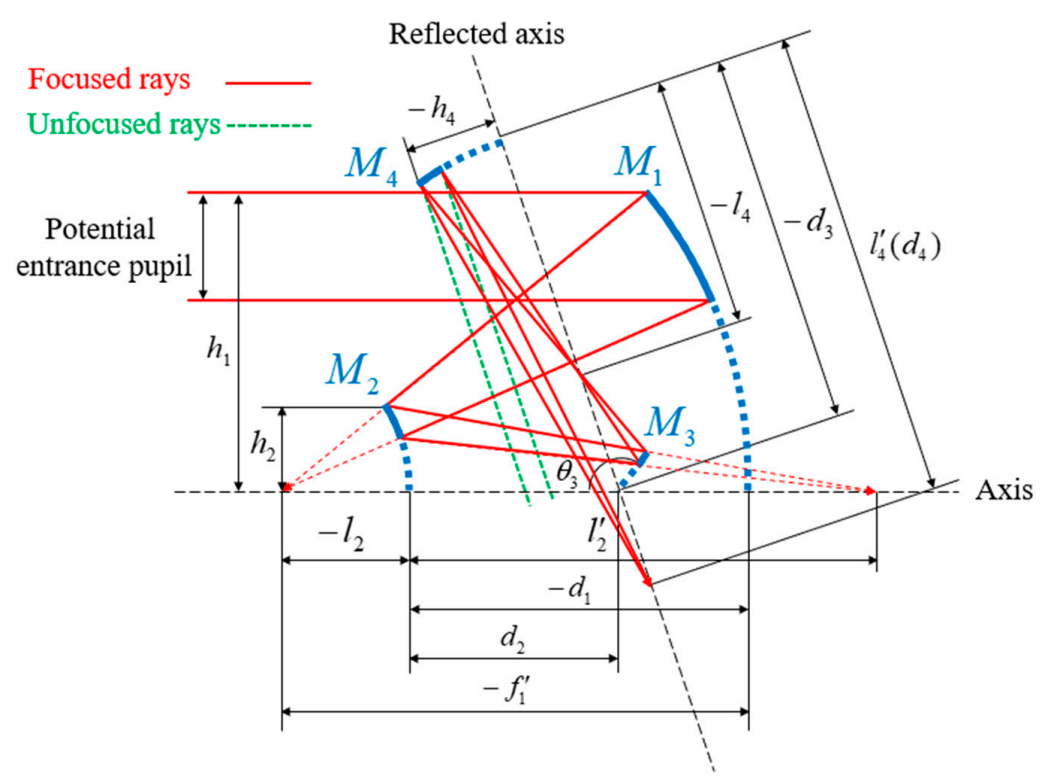

Figure 5. The layout of the four-mirror system.

\subsection{Search for the Initial Structure via GA}

GA is a global optimization algorithm that does not depend on the initial parameters. It uses the principle of nature to "evolve" toward an optimal solution. For highly nonlinear and high-dimensional parameter optimization problems, GA can often find the optimal solution effectively [28]. In our work, GA is used to optimize the objective function to obtain the initial optical system parameters. The algorithm iteratively optimizes the objective function by relying on biologically inspired operators such as mutation, crossover and selection. 
Based on the analyses of the manufacturing constraints, aberrations and the structure configuration in the previous section, the objective function was established.

The objective function of aberrations is composed of weighted aberrations which can be expressed as $f_{a}$,

$$
f_{a}=f_{a}\left(w_{i}, \alpha_{1}, \alpha_{2}, \beta_{1}, \beta_{2}, k_{1}, k_{2}, k_{4}\right)=w_{1}\left|S_{1}\right|+w_{2}\left|S_{2}\right|+w_{3}\left|S_{3}\right|+w_{4}\left|S_{4}\right|+w_{5}\left|S_{5}\right|,
$$

where $w_{i}(i=1,2 \ldots 5)$ is the weight of the corresponding term. The weight given to each term depends on its importance in the system. The higher weight values are set to the aberration coefficients with high requirements and vice versa.

According to Equation (10), the objective function $f_{a}$ is a comprehensive reflection of the aberration in the optical system. The smaller $f_{a}$ value indicates a better imaging quality of the optical system.

The objective function of manufacturing and structural constraints $f_{c}$ consists of the ADRS, the tool spindle path $C$ and some other structural constraints $O$, such as the obscuration elimination and telecentric in the image space, are as follows:

$$
f_{c}=f_{c}\left(w_{i}, \alpha_{1}, \alpha_{2}, \beta_{1}, \beta_{2}, k_{1}, k_{2}, k_{4}, d_{2}, \theta_{3}\right)=w_{6}\left|\sigma_{\mathrm{ADRS}}\right|+w_{7}|C|+w_{i}|O|,
$$

where $d_{2}$ and $\theta_{3}$ are the additional variables added for structure control.

The manufacturing and structural constraints function $f_{c}$ and imaging quality constraints function $f_{a}$ constitute the objective function $F$ :

$$
F=f_{a}+f_{c} .
$$

By establishing the objective function, the problem of solving the initial structural parameters of the four-mirror optical system is transformed into the optimization of the objective function $F$. The small value of aberration coefficients, ADRS and tool spindle path $C$ will be obtained by minimizing $F$. In this paper, GA is introduced to optimize the objective function, that is, GA is introduced to solve the parameters of the initial structure.

The optimization process of GA is shown in Figure 6, which is briefly described as follows:

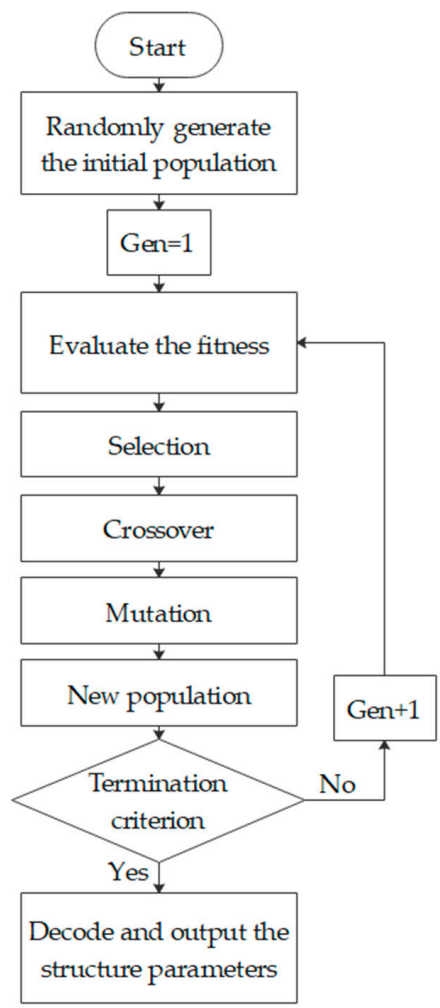

Figure 6. Flowchart of the genetic algorithm (GA) process. 
Step 1: Encode the parameters and initialize the population. Encoding is the basis of GA, and the encoding mechanism has an essential influence on the performance and efficiency of the algorithm. In this paper, binary is used to encode the parameters [29]. First, convert parameters $\alpha_{1}, \alpha_{2}, \beta_{1}, \beta_{2}, k_{1}$, $k_{2}, k_{4}, d_{2}$, and $\theta_{3}$ from decimal numbers to binary numbers. The sequence of 9 parameters represents a chromosome, which is a solution of the objective function. Each generation consists of a certain amount of chromosomes, and the population size is set empirically. Then, the initial population of GA is formed by randomly generating multiple chromosomes.

Step 2: Evaluate the fitness. The reciprocal of the objective function is used as the fitness function to calculate the fitness of each chromosome in the population. The value of fitness is the main performance index to describe the performance of an individual in GA. The larger fitness value indicates a good individual's performance and vice versa.

Step 3: Selection. Once the fitness is calculated, several pairs of chromosomes were selected as parents for breeding. The chromosome with a larger value of fitness has a higher selection probability.

Step 4: Crossover. Crossover is the operator that allows selected chromosomes to exchange some genes with the crossover probability $p_{c}$, which is an important means to obtain excellent individuals in GA. The $p_{c}$ is set between 0.8 and 1.0. In binary coding, crossover methods include single point crossover, two-point crossover and multi-point crossover.

Step 5: Mutation. Mutation is the operation of changing some genes of a chromosome to form new individuals with a certain probability (mutation probability). For the mutation in binary, the chromosomes are mutated at one point selected randomly. The mutation of bit is the inversion of bit: 0 becomes 1 or 1 becomes 0 . In GA, mutation is employed to avoid converging to local minima. The mutation probability $p_{m}$ is approximately 0.01 .

Step 6: After finishing the process of crossover and mutation operations, some new individuals are produced to join the surviving individuals so as to form a new generation.

Step 7: Termination conditions. The termination condition is usually set as the maximum number of generations or no obvious changes in the value of the objective function.

Step 8: Decode and calculate the initial structure parameters $\alpha_{1}, \alpha_{2}, \beta_{1}, \beta_{2}, k_{1}, k_{2}, k_{4}, d_{2}$, and $\theta_{3}$.

The flow chart of the whole design process is shown in Figure 7, which is described as follows:

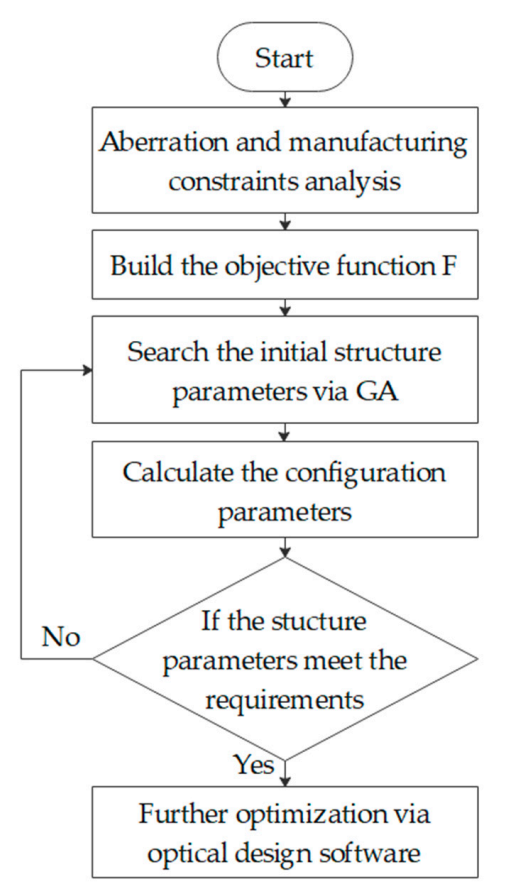

Figure 7. Flowchart of the design process. 
Step 1: Establish the objective function $F=f\left(w_{i}, \alpha_{1}, \alpha_{2}, \beta_{1}, \beta_{2}, k_{1}, k_{2}, k_{4}, d_{2}, \theta_{3}\right)$ by analyzing the aberrations and manufacturing constraints.

Step 2: Set the weights and use GA to optimize the parameters $\left(w_{i}, \alpha_{1}, \alpha_{2}, \beta_{1}, \beta_{2}, k_{1}, k_{2}, k_{4}, d_{2}, \theta_{3}\right)$ to minimize the objective function.

Step 3: Based on the optimized parameters in the previous step, calculate the configuration parameters $R_{1}, R_{2}, R_{4}, d_{1}, d_{3}$, and $d_{4}$.

Step 4: The configuration parameters were imported for further analysis into the commercial software, and then, the final system is obtained.

In this study, the objective function consists of two parts: the aberration constraints and the manufacturing constraints, in which the calculation of manufacturing constraints is very time-consuming because of the ray tracing. This phenomenon is more obvious when the population size and the number of iterations is large.

In order to solve this problem, here we propose a strategy to speed up the calculation. The fitness calculation process of the GA is improved as shown in the flow chart in Figure 8. In this improved strategy, the aberration constraints $f_{a}$ is first calculated instead of the objective function $F$, and then the value of $f_{a}$ is compared with the threshold. The inferior/poor individuals will directly use the $f_{a}^{-1}$ as the fitness to implement the selection operation without calculating manufacturing constraints $f_{c}$; As for the excellent individuals, $f_{c}^{-1}$ will be calculated and added to $f_{a}^{-1}$ to make them more competitive, which is more like an incentive process. As the iteration goes on, the proportion of individuals with small aberrations and good structural configurations will increase in the population.

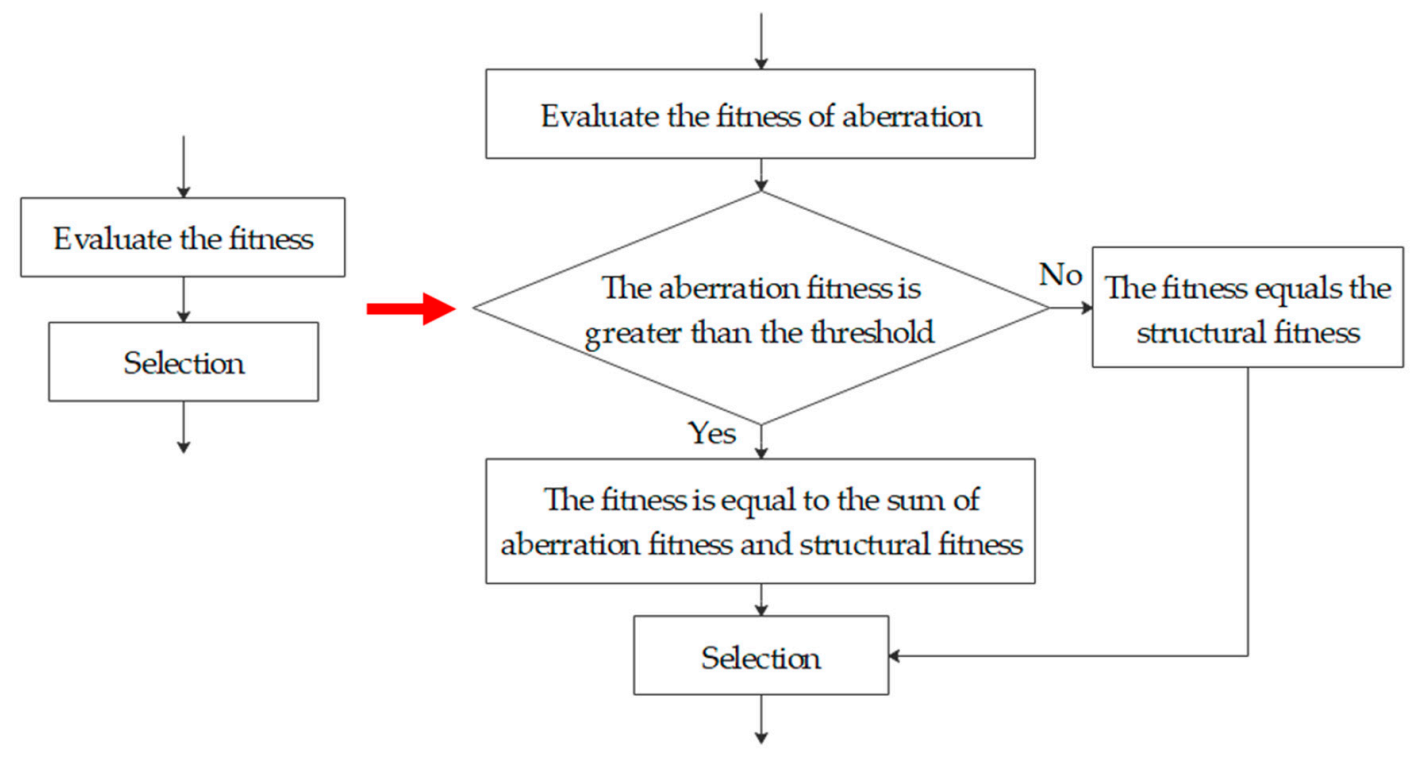

Figure 8. Improvement of the fitness calculation.

\section{Design Approach}

In this section, an off-axis four-mirror afocal reflective system, which is satisfying manufacturing constraints, is presented as an example. Table 1 shows the specification of the system.

Table 1. Specification of the off-axis four-mirror optical system.

\begin{tabular}{cc}
\hline Parameter & Specification \\
\hline Entrance pupil diameter/mm & 170 \\
Exit pupil diameter/mm & 34 \\
Field of view $/\left(^{\circ}\right)$ & $3 \times 3$ \\
Wavelength $/ \mathrm{nm}$ & $650 \sim 900$ \\
Compression ratio & 5 \\
\hline
\end{tabular}


According to the method proposed in Section 2, the GA program is written to calculate the initial structural parameters of the off-axis four-mirror system. The main parameters of GA are the population size $n=50, p_{c}=0.9, p_{m}=0.01$, and evolutionary generations are 200 .

The objective function is composed of primary aberration coefficients and structural layout constraints with weights

$$
\begin{aligned}
& F=f\left(w_{i}, \alpha_{1}, \alpha_{2}, \beta_{1}, \beta_{2}, k_{1}, k_{2}, k_{4}, d_{2}, \theta_{3}\right) \\
& =w_{1}\left|S_{1}\right|+w_{2}\left|S_{2}\right|+w_{3}\left|S_{3}\right|+w_{4}\left|S_{4}\right|+w_{5}\left|S_{5}\right|+w_{6}\left|\sigma_{\mathrm{ADRS}}\right|+w_{7}|C|+w_{i}|O| .
\end{aligned}
$$

Certain conditions are set to obtain reasonable initial structural parameters: $\alpha_{1}>0, \alpha_{2}<0, \beta_{1}<0$ and $\beta_{2}>0$. In this case, we know that there is an intermediate image plane in this structure. In addition, these parameters need to satisfy some boundary conditions, which are shown in Table 2.

Table 2. Parameters range.

\begin{tabular}{cccccccccc}
\hline Parameter & $\alpha_{1}$ & $\alpha_{2}$ & $\beta_{1}$ & $\beta_{2}$ & $k_{1}$ & $k_{2}$ & $k_{4}$ & $d_{2}(\times 10)$ & $\boldsymbol{\theta}_{3}$ \\
\hline Range & $(0,0.5)$ & $(-1,0)$ & $(-5,0)$ & $(0,5)$ & $(0,5)$ & $(0,5)$ & $(0,5)$ & $(42,45)$ & $(1.6,2)$ \\
\hline
\end{tabular}

According to the 9 parameters with ranges, GA is used to optimize the objective function $F$ mentioned before.

Figure 9 shows the convergence curve of the objective function. The data point on the curve indicates the elitist individual of each generation. It can be seen that the value of the objective function decreases as iteration times increase, and the objective function is convergent.

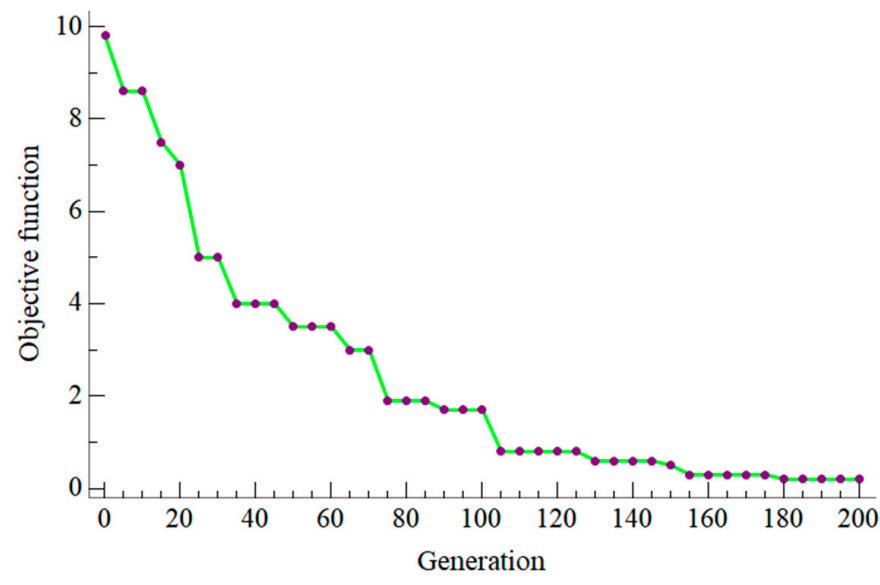

Figure 9. Convergence curve of the objective function.

Multiple groups of initial structure parameters have been obtained by changing the weight. Five groups of data with the lowest convergent $F$ values are listed in Table 3. The optimization results of each group are close, which proves the stability of the GA in dealing with optical optimization with special requirements.

Table 3. Initial structures parameters obtained for the off-axis four-mirror system.

\begin{tabular}{cccccccccc}
\hline No. & $\boldsymbol{\alpha}_{1}$ & $\boldsymbol{\alpha}_{2}$ & $\boldsymbol{\beta}_{1}$ & $\boldsymbol{\beta}_{2}$ & $\boldsymbol{k}_{1}$ & $\boldsymbol{k}_{2}$ & $\boldsymbol{k}_{4}$ & $\boldsymbol{d}_{2}$ & $\boldsymbol{\theta}_{3}$ \\
\hline 1 & 0.300 & -0.742 & -2.360 & 4.126 & 0.867 & 4.127 & 0.780 & 438.27 & 1.89 \\
2 & 0.270 & -0.661 & -2.881 & 4.354 & 0.963 & 3.849 & 0.800 & 430.26 & 1.90 \\
3 & 0.275 & -0.900 & -2.381 & 4.086 & 0.896 & 3.907 & 0.685 & 434.96 & 1.84 \\
4 & 0.257 & -0.862 & -2.456 & 4.094 & 0.876 & 3.484 & 0.733 & 434.43 & 1.87 \\
5 & 0.293 & -0.856 & -2.269 & 4.315 & 0.790 & 2.804 & 0.742 & 436.24 & 1.83 \\
\hline
\end{tabular}


According to the Equation (7), the structural parameters are calculated and listed in Table 4.

Table 4. Configuration parameters of the initial off-axis four-mirror system.

\begin{tabular}{cccc}
\hline No. & $\boldsymbol{R}_{1}(\mathbf{m m})$ & $\boldsymbol{R}_{2}(\mathbf{m m})$ & $\boldsymbol{R}_{4}(\mathbf{m m})$ \\
\hline 1 & -1817.14 & -946.21 & 768.66 \\
2 & -1742.47 & -720.60 & 728.13 \\
3 & -1764.08 & -810.99 & 810.15 \\
4 & -1711.21 & -740.85 & 747.52 \\
5 & -1791.55 & -928.71 & 819.44 \\
\hline
\end{tabular}

A larger $\alpha_{1}$ value can make the optical system structure more compact, so the first structure in Table 3 is selected as the initial structure for further optimization in software. The ADRS and the maximum deviation from the reference surface of each mirror in the initial structure are listed in Table 5.

Table 5. The average deviation from the reference surface (ADRS) and the maximum deviation of each mirror in the initial structure.

\begin{tabular}{ccccc}
\hline No. & M1 & M2 & M3 & M4 \\
\hline ADRS (mm) & 4.23 & 5.71 & 8.49 & 3.88 \\
Maximum deviation $(\mathrm{mm})$ & 13.74 & 11.40 & 15.54 & 4.11 \\
\hline
\end{tabular}

Figure 10 shows the layouts of the initial afocal structure with parameters calculated by GA, in which the red circle with a radius of $337 \mathrm{~mm}$ represents the reference surface. Figure 11 shows the layouts of the structure of which few of FOVs have been optimized. The modulation transfer function (MTF) curves for the initial and optimized structures are also presented in Figures 12 and 13, respectively. The MTF curves show that each system is close to the diffraction limit, indicating that the initial structure obtained from GA is a good starting point for further optimization.

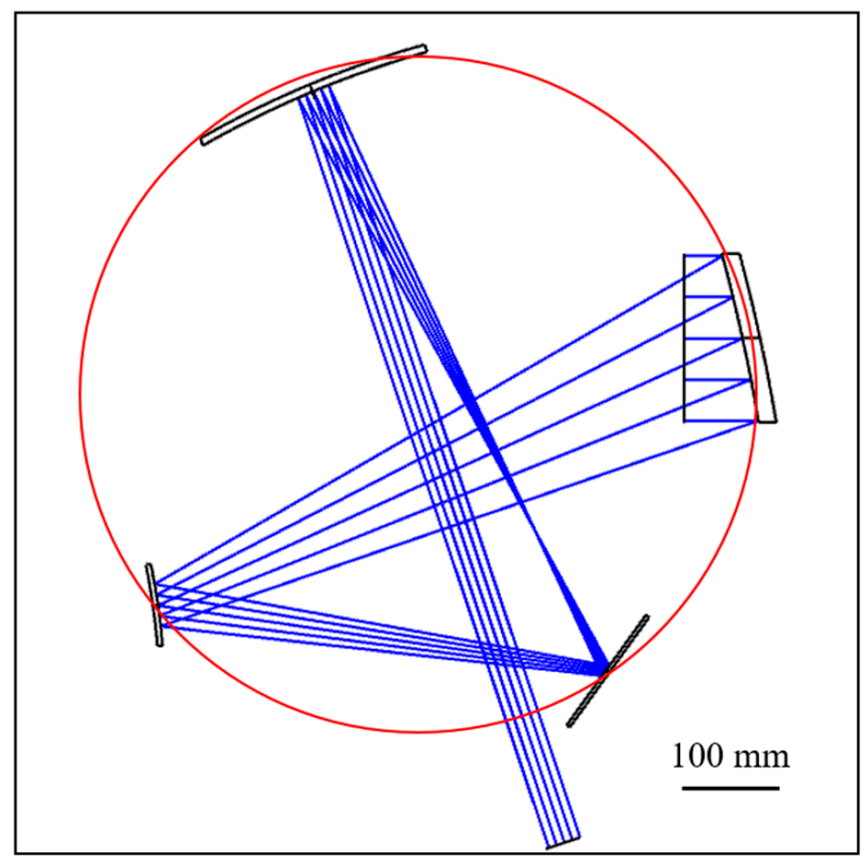

Figure 10. Initial structure layout of the off-axis four-mirror system. 


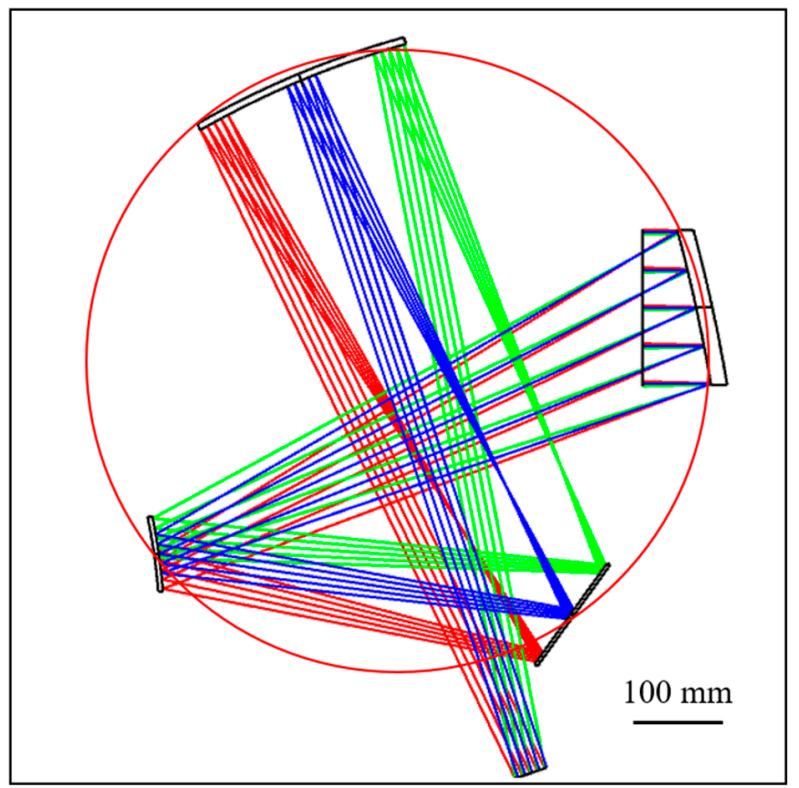

Figure 11. Optimized structure layout of the off-axis four-mirror system.

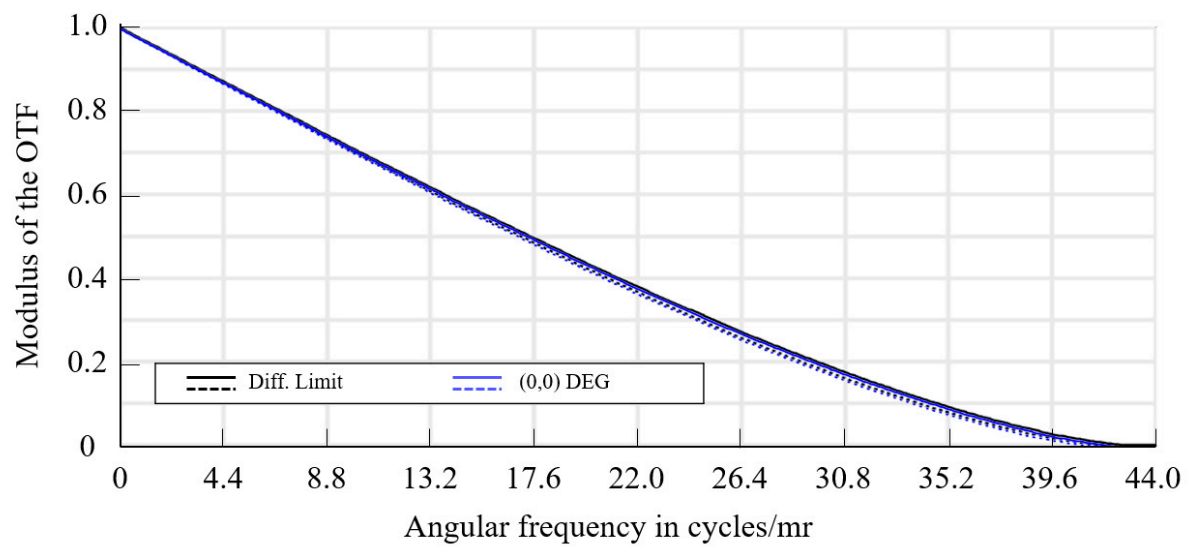

Figure 12. Initial structure modulation transfer function (MTF) of the off-axis four-mirror system.

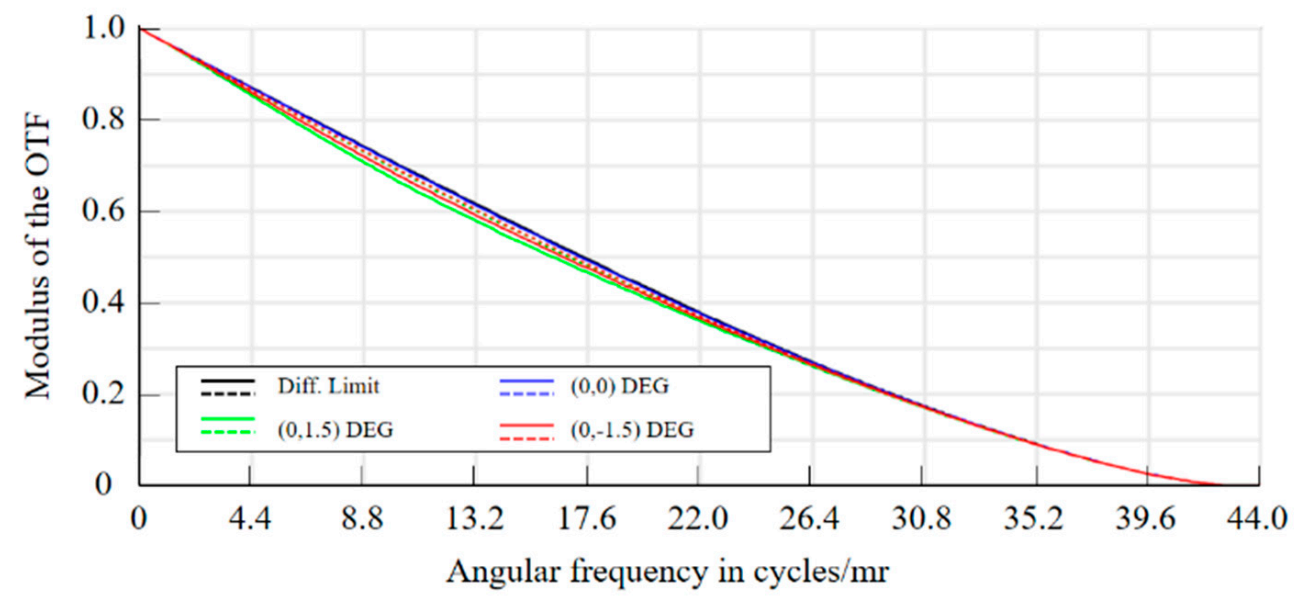

Figure 13. Optimized structure MTF of the off-axis four-mirror system. 
The configuration parameters of the optimized structure are presented in Table 6. After further optimization, the structural parameters are still close to the initial structure, which proves that it is feasible to use GA to find the initial structure with special requirements.

Table 6. Configuration parameters of the optimized off-axis four-mirror system.

\begin{tabular}{cccc}
\hline Mirror & $\mathbf{R}(\mathbf{m m})$ & $\mathbf{d}(\mathbf{m m})$ & Conic \\
\hline Primary & -1947.38 & -636.00 & 1.020 \\
Secondary & -865.21 & 438.27 & 4.297 \\
Quaternary & 964.89 & 820.00 & 0.942 \\
\hline
\end{tabular}

In the next step, the freeform surface is introduced to further improve the imaging quality [30].

The freeform surface is a category of non-rotational symmetric surfaces. Compared with the traditional optical surface such as spherical and aspheric surfaces, it has a stronger ability to correct aberrations. With the development of manufacturing technology, freeform surfaces are more and more used in illumination and imaging systems.

In this structure, we choose the Zernike polynomial surface to optimize the mirrors [31]. Zernike polynomials can correspond to Seidel aberration coefficients in optical design.

The Zernike polynomial surface is composed of the conical surface and additional aspheric terms defined by the Zernike polynomial coefficients. The Fringe ordering of the Zernike polynomials is chosen, with sag described mathematically by

$$
z=\frac{c r^{2}}{1+\sqrt{1-(1+k) c^{2} r^{2}}}+\sum_{i=1}^{N} C_{i} Z_{i}(\rho, \varphi),
$$

where $c$ is the curvature of the base sphere, $r$ is the radial coordinate of the surface, $k$ is the conic constant, $\rho$ is the radial coordinate of the surface normalized by $R_{\text {norm }}$ (that is, $\rho=r / R_{\text {norm }}$ ), $\varphi$ is the azimuthal component of the surface aperture, and $C_{i}$ is the weight factor of the $i^{\text {th }}$ Zernike term, $Z_{i}$.

After setting the tertiary mirror to the Zernike surface, the Aspheric surface high-order coefficients and Zernike coefficients of the tertiary mirror are shown in Table 7, and the configuration parameters of the final off-axis four-mirror system are shown in Table 8.

Table 7. Aspheric surface high-order coefficients and Zernike coefficients of the tertiary mirror.

\begin{tabular}{ccccc}
\hline Mirror & $\begin{array}{c}\text { Fourth Order } \\
\text { Coefficient }\end{array}$ & $\begin{array}{c}\text { Sixth Order } \\
\text { Coefficient }\end{array}$ & $\begin{array}{c}\text { Zernike Third } \\
\text { Coefficient }\end{array}$ & $\begin{array}{c}\text { Zernike Fifth } \\
\text { Coefficient }\end{array}$ \\
\hline Tertiary & $-1.810 \times 10^{-10}$ & $-7.727 \times 10^{-15}$ & $6.6 \times 10^{-4}$ & $-1.506 \times 10^{-5}$ \\
\hline
\end{tabular}

Table 8. Configuration parameters of the final off-axis four-mirror system.

\begin{tabular}{cccccc}
\hline Mirror & $\mathbf{R}(\mathbf{m m})$ & $\mathbf{d}(\mathbf{m m})$ & Conic & Fourth Order Coefficient & Sixth Order Coefficient \\
\hline Primary & -1682.54 & -636.00 & 1.003 & $-1.039 \times 10^{-13}$ & $-5.334 \times 10^{-20}$ \\
Secondary & -615.69 & 438.27 & 4.039 & $-2.681 \times 10^{-11}$ & $-1.514 \times 10^{-16}$ \\
Quaternary & 1009.27 & 820.00 & 0.986 & $-4.901 \times 10^{-12}$ & $-1.796 \times 10^{-17}$ \\
\hline
\end{tabular}

The final structure layout of the off-axis four-mirror system is shown in Figure 14, in which the rays tracing of all FOVs are plotted with different colors. All mirrors in the final structure are distributed approximately along a cylinder. 


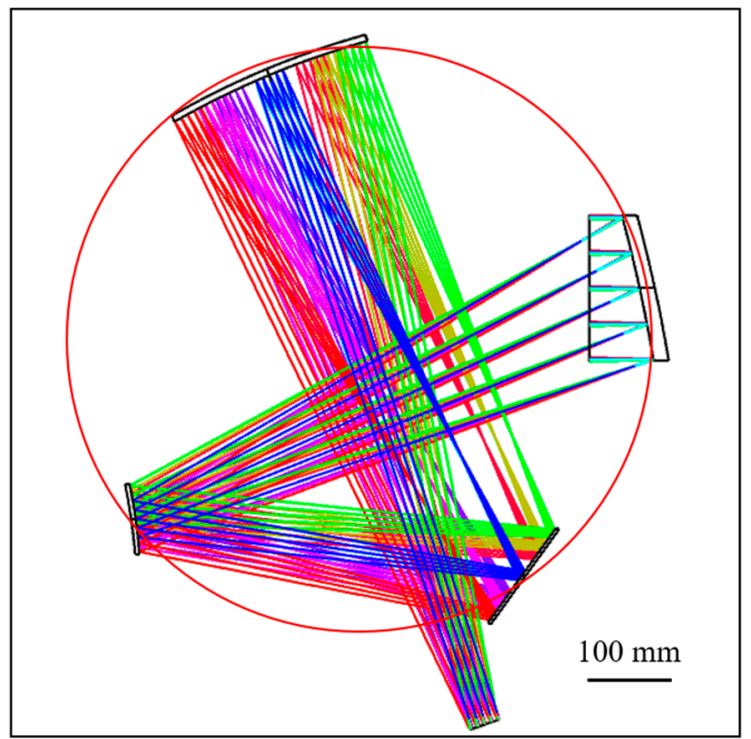

Figure 14. Final structure layout of the off-axis four-mirror system.

The ADRS and the maximum deviation from the reference surface of each mirror in the final structure are listed in Table 9. The ADRS values show that mirrors in the system are close to the reference surface.

Table 9. The ADRS and the maximum deviation of each mirror in the final structure.

\begin{tabular}{ccccc}
\hline No. & M1 & M2 & M3 & M4 \\
\hline ADRS (mm) & 4.78 & 5.16 & 7.34 & 3.42 \\
Maximum deviation $(\mathrm{mm})$ & 13.08 & 13.75 & 14.05 & 5.18 \\
\hline
\end{tabular}

The modulation transfer function (MTF) of the system is shown in Figure 15. The MTFs at 13 cycles/mr are above 0.6 for all FOVs, which indicates that the system has a good optical performance.

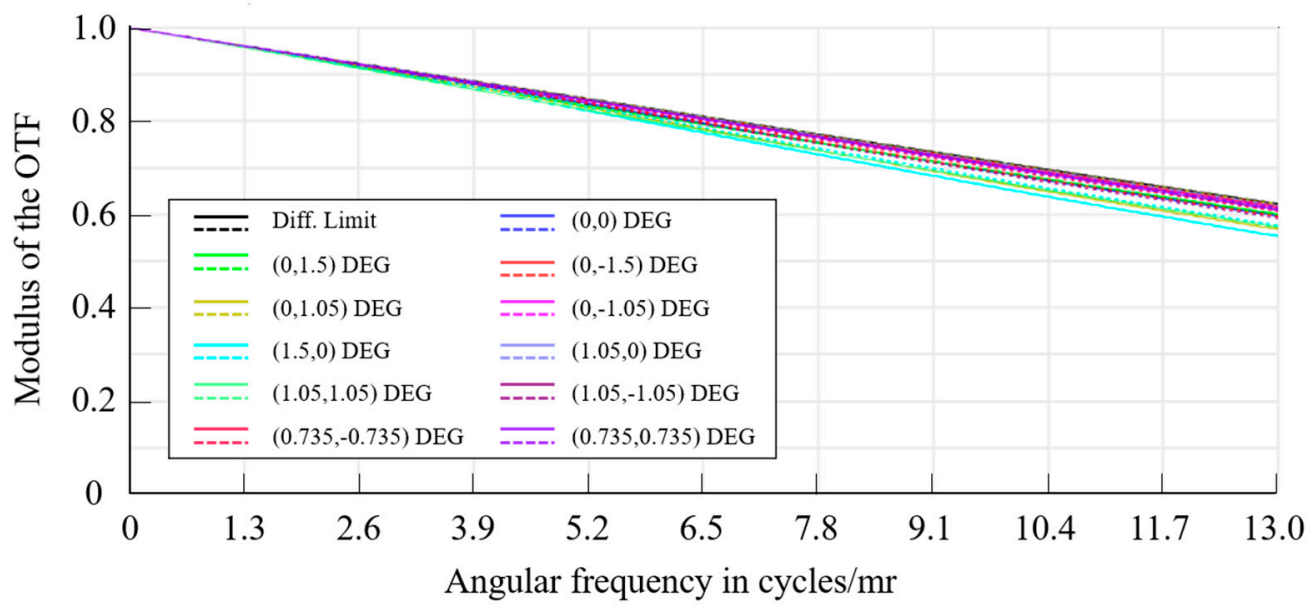

Figure 15. Final structure MTF of the off-axis four-mirror system.

Figure 16a shows the gird distortion of the final off-axis four-mirror system. The paraxial FOV grid coincides with the actual FOV grid approximately, which indicates that there is no obvious distortion in the system. Figure 16b shows the F-Tan (Theta) distortion of the system. The distortion increases gently as the FOV increases and the maximum distortion is approximately $0.4 \%$. 


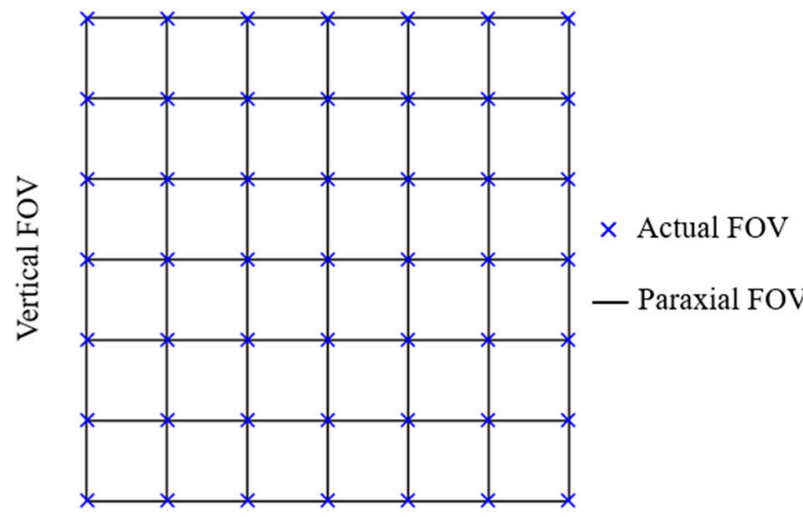

Horizontal FOV

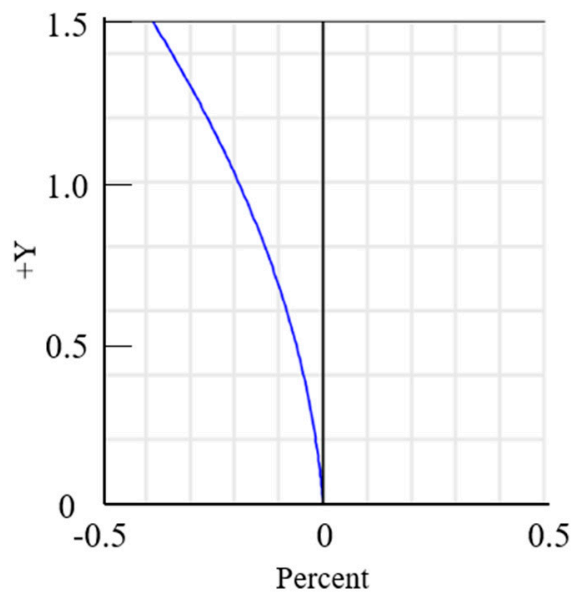

(b)

Figure 16. Distortion of the off-axis four-mirror system. (a) Grid distortion. (b) F-Tan (Theta) distortion.

\section{Conclusions}

The introduction of manufacturing constraints in the optical design process can guarantee the optical performance of the system and facilitate the assembly and machining process, which is beneficial to further develop the applications of optical systems.

In this paper, a novel method for finding the initial structure parameters of an off-axis four-mirror reflective system considering manufacturing constraints via GA is proposed. In this method, in order to find the appropriate initial structural parameters, the GA is employed to optimize the objective function, which is established according to aberration analyses and manufacturing constraints analyses. The GA is computationally efficient when finding the global minima in complex, highly nonlinear and high-dimensional parameter spaces. In addition, the reasonable definition of the objective function and the optimization strategy ensure the possibility of using GA successfully. This method helps solve the complex optical alignment problem in optical systems. The off-axis four mirror system designed according to this method can be put into use without optical alignment once the machining is completed. This method is also applicable to off-axis three-mirror systems, off-axis five-mirror systems and coaxial reflective systems.

The feasibility of the proposed method is validated by designing an off-axis four-mirror afocal system with an entrance pupil diameter of $170 \mathrm{~mm}$, a field of view of $3^{\circ} \times 3^{\circ}$, an operating wave band of 650 900 nm, and a compression ratio of five times, in which all the mirrors are guaranteed to be distributed along a cylinder $337 \mathrm{~mm}$ in radius to facilitate the ultraprecise raster milling. The results show that the optimization method based on GA can provide a good starting point for the design of the reflective optical system with special requirements. In the future, by integrating this method with different optical structures, a synthetic method to deal with the design of manufacturing-constrained optical systems can be obtained.

Author Contributions: Conceptualization, Z.L. and F.F.; methodology, R.L.; software, R.L.; validation, R.L.; formal analysis, R.L. and Z.L.; investigation, R.L., Z.L. and Y.D.; resources, F.F.; data curation, R.L.; writing-original draft preparation, R.L.; writing - review and editing, R.L., Z.L., Y.D. and F.F.; visualization, R.L.; supervision, F.F.; project administration, F.F.; funding acquisition, F.F. All authors have read and agreed to the published version of the manuscript.

Funding: This research was funded by the National Key Research and Development Program of China (Grant No. 2017YFA0701200), the Postdoctoral Innovative Talent Support Program of China (BX20190230), the National Natural Science Foundation (No. 61635008), and the "111" project conducted by the State Administration of Foreign Experts Affairs and the Ministry of Education of China (No. B07014) 
Acknowledgments: The authors express their sincere thanks to Ran Hao from Tianjin University for valuable discussions.

Conflicts of Interest: The authors declare no conflict of interest.

\section{References}

1. Metwally, M.; Bazan, T.M.; Eltohamy, F. Design of Very High-Resolution Satellite Telescopes Part I: Optical System Design. IEEE Trans. Aerosp. Electron. Syst. 2020, 56, 1202-1208. [CrossRef]

2. Ye, J.; Yu, J.; Song, Z.; Pei, S.; Yuan, Q.; Gao, Z.; He, Y. Design of a compact off-axis two-mirror freeform infrared imager with a wide field of view. J. Mod. Opt. 2018, 66, 304-311. [CrossRef]

3. Marinelli, W.J.; Gittins, C.M.; Gelb, A.H.; Green, B.D. Tunable Fabry-Perot etalon-based long-wavelength infrared imaging spectroradiometer. Appl. Opt. 1999, 38, 2594. [CrossRef]

4. Meng, Q.; Wang, H.; Liang, W.; Yan, Z.; Wang, B. Design of off-axis three-mirror systems with ultrawide field of view based on an expansion process of surface freeform and field of view. Appl. Opt. 2019, 58, 609-615. [CrossRef]

5. Fang, F.; Zhang, X.; Weckenmann, A.; Zhang, G.; Evans, C. Manufacturing and measurement of freeform optics. CIRP Ann. Manuf. Technol. 2013, 62, 823-846. [CrossRef]

6. Fang, F.; Xu, F. Recent Advances in Micro/Nano-cutting: Effect of Tool Edge and Material Properties. Nanomanuf. Metrol. 2018, 1, 4-31. [CrossRef]

7. Burada, D.R.; Pant, K.K.; Mishra, V.; Bichra, M.; Khan, G.S.; Sinzinger, S.; Shakher, C. Development of a metrology technique suitable for in situ measurement and corrective manufacturing of freeform optics. Adv. Opt. Technol. 2019, 8, 203-215. [CrossRef]

8. Meng, Q.; Wang, W.; Ma, H.; Dong, J. Easy-aligned off-axis three-mirror system with wide field of view using freeform surface based on integration of primary and tertiary mirror. Appl. Opt. 2014, 53, 3028-3034. [CrossRef]

9. Li, Z.; Liu, X.; Fang, F.; Zhang, X.; Zeng, Z.; Zhu, L.; Yan, N. Integrated manufacture of a freeform off-axis multi-reflective imaging system without optical alignment. Opt. Express 2018, 26, 7625-7637. [CrossRef]

10. Zheng, X.; Li, Z.; Zhang, X.; Fang, F. Manufacturing-constrained optical design methodology for cylindrical freeform reflective imaging system. Opt. Express 2018, 26, 22547-22562. [CrossRef]

11. Li, Z.; Fang, F.; Chen, J.; Zhang, X. Machining approach of freeform optics on infrared materials via ultra-precision turning. Opt. Express 2017, 25, 2051-2062. [CrossRef] [PubMed]

12. Zhang, X.; Gao, H.; Guo, Y.; Zhang, G. Machining of optical freeform prisms by rotating tools turning. CIRP Ann. Manuf. Technol. 2012, 61, 519-522. [CrossRef]

13. Brecher, C.; Lange, S.; Merz, M.; Niehaus, F.; Wenzel, C.; Winterschladen, M.; Weck, M. NURBS Based Ultra-precision Free-form Machining. CIRP Ann. Manuf. Technol. 2006, 55, 547-550. [CrossRef]

14. Li, L.; Xu, X.-Q.; Wang, Q.-H. Mutated and converged damping factors in lens system optimization to find new local minima. Optik 2013, 124, 4150-4154. [CrossRef]

15. Wei, L.; Hanchun, Y.; Baoping, W.; Linsu, T. Optimization of a particle optical system in a mutilprocessor environment. Nucl. Instrum. Methods Phys. Res. Sect. A Accel. Spectrometers Detect. Assoc. Equip. 2002, 479, 611-617. [CrossRef]

16. Whitley, D. A genetic algorithm tutorial. Stat. Comput. 1994, 4, 65-85. [CrossRef]

17. Mitchell, M. An Introduction to Genetic Algorithms; MIT Press: Cambridge, MA, USA, 1998.

18. Jun, L.; Huang, W.; Hongiie, F. A novel method for finding the initial structure parameters of optical systems via a genetic algorithm. Opt. Commun. 2016, 361, 28-35. [CrossRef]

19. Chatterjee, S.; Hazra, L. Structural design of cemented triplets by genetic algorithm. Opt. Eng. 2004, 43, 432-440. [CrossRef]

20. Höschel, K.; Lakshminarayanan, V. Genetic algorithms for lens design: A review. J. Opt. 2018, 48, $134-144$. [CrossRef]

21. Cao, C.; Liao, S.; Liao, Z.; Bai, Y.; Fan, Z. Initial configuration design method for off-axis reflective optical systems using nodal aberration theory and genetic algorithm. Opt. Eng. 2019, 58, 105101. [CrossRef]

22. Zhou, G.; Chen, Y.; Wang, Z.; Song, H. Genetic local search algorithm for optimization design of diffractive optical elements. Appl. Opt. 1999, 38, 4281-4290. [CrossRef] [PubMed] 
23. Yan, F.; Zhang, X. Optimization of an off-axis three-mirror anastigmatic system with wavefront coding technology based on MTF invariance. Opt. Express 2009, 17, 16809-16819. [CrossRef] [PubMed]

24. Feng, Q.; Yang, F.; Xu, X.; Zhang, B.; Ding, Y.; Liu, Q. Multi-objective optimization genetic algorithm for multi-point light focusing in wavefront shaping. Opt. Express 2019, 27, 36459-36473. [CrossRef] [PubMed]

25. Mignard-Debise, L. Tools for the Paraxial Optical Design of Light Field Imaging Systems. Ph.D. Thesis, Université de Bordeaux, Bordeaux, France, 2018.

26. Terebizh, V.Y. Algorithm for calculating anastigmatic three-mirror telescopes. Exp. Astron. 2020, 49, 85-95. [CrossRef]

27. Junhua, P. The Design, Manufacture and Test of the Aspherical Optical Surfaces; Publishing of Suzhou University Press: Suzhou, China, 2004.

28. Rao, S.S. Engineering Optimization: Theory and Practice; John Wiley \& Sons: Hoboken, NJ, USA, 2019.

29. Bridges, C.L.; Goldberg, D.E. An analysis of reproduction and crossover in a binary-coded genetic algorithm. Grefenstette 1987, 878, 9-13.

30. Fang, F.; Cheng, Y.; Zhang, X. Design of freeform optics. Adv. Opt. Technol. 2013, 2, 445-453. [CrossRef]

31. Khonina, S.N.; Karpeev, S.V.; Porfirev, A.P. Wavefront Aberration Sensor Based on a Multichannel Diffractive Optical Element. Sensors 2020, 20, 3850. [CrossRef]

(C) 2020 by the authors. Licensee MDPI, Basel, Switzerland. This article is an open access article distributed under the terms and conditions of the Creative Commons Attribution (CC BY) license (http://creativecommons.org/licenses/by/4.0/). 